\title{
A new version of smoothed point method to simulate linear elastic wave propagation
}

\author{
Hua Ding *, Yan-Feng Wu, Li-Ping Long, Nai-Gang Liang \\ Institute of Mechanics, CAS, 100080 Beijing, China
}

Available online 15 June 2005

\begin{abstract}
By using the kernel function of the smoothed particle hydrodynamics ( $\mathrm{SPH}$ ) and modification of statistical volumes of the boundary points and their kernel functions, a new version of smoothed point method is established for simulating elastic waves in solid. With the simplicity of SPH kept, the method is easy to handle stress boundary conditions, especially for the transmitting boundary condition. A result improving by de-convolution is also proposed to achieve high accuracy under a relatively large smooth length. A numerical example is given and compared favorably with the analytical solution.
\end{abstract}

(c) 2005 Elsevier Ltd. All rights reserved.

Keywords: SPH; Very weak estimates; Elastic wave propagation; Transmitting boundary condition; De-convolution conversion

\section{Introduction}

Smoothed particle hydrodynamics (SPH) offered a meshless, Lagrangian particle method for modeling fluid flows. It is a truly meshless method in computational continuum dynamics. Since it was proposed in 1977 (Lucy, 1977; Gingold and Monaghan, 1977), the method has been applied to astrophysical and cosmological problems throughout the decade of the 1980s and to the present. In 1991, SPH was extended to treat the dynamic response of solids (Libersky and Petschek, 1991) and from then, such a method has been applied more and more widely in new and interesting fields, e.g. the studies on constructions of the kernel function (Liu et al., 2003a), ataptive SPH methods (Owen et al., 1998), and applications to high explosive explosion (Liu et al., 2003b,c), under water explosion (Liu et al., 2003d), water migration (Liu et al., 2002) etc., also some improvements has been done with stress points (Randles and Libersky, 2000). Recently a

\footnotetext{
* Corresponding author. Tel.: +86162657887; fax: +86162561284.

E-mail address: hding@imech.ac.cn (H. Ding).
} 
comprehensive book on the basic theory, recent development and trend of the SPH method has been published (Liu and Liu, 2003).

Continuum dynamics is governed by partial differential equations, but in SPH the field information is condensed onto discrete points in the field. The partial differential equations are transformed into integral equations through an interpolation function as a 'kernel estimate' of the field variables at the discrete points. The integrals are evaluated as sums over neighboring particles. As long as the values at those particle points are obtained, field information can be calculated by interpolation from the values at the particle points without need of any underlying grid as that in FEM.

In SPH and allied methods, an arbitrary function $f(x)$ will be reproduced to be its kernel approximation in terms of a kernel function $W(x, h)$ as

$$
\langle f\rangle_{h}(x)=\int f(y) W(x-y, h) \mathrm{d} y
$$

where $h$ is the smoothing length (Lucy, 1977; Gingold and Monaghan, 1977).

Clearly, the reproduced function $\langle f\rangle_{h}(x)$ will converge to the exact function $f(x)$ as $W(x, h)$ approaches the Dirac delta function $\delta(x)$. Further, by proper selection of this kernel function, it is able to ensure that polynomials up to a given degree $k$ are exactly reproduced by the above integral. If the selected $W(x, h)$, taken one-dimensional problem as example, meet

$$
\int W(x, h) \mathrm{d} x=1, \quad \int x^{j} W(x, h) \mathrm{d} x=0, \quad 0<j \leqslant k,
$$

the approximation is said to be of order $k$ and $\langle f\rangle_{h}(x)$ will coincide with $f(x)$ for polynomials of up to $k$ degrees. Eq. (2) is often referred to as consistency condition. Since smoothing length $h$ does not affect on formulation of the algorithm, it will be omitted in the followings when there is no confusion, e.g., the kernel function $W(x, h)$ will be written as $W(x)$ until Section 7. For numerical purpose, the approximation of $f(x)$ given in Eq. (1) is evaluated with

$$
\langle f\rangle(x)=\sum_{b \in M_{x}} f\left(x^{b}\right) W\left(x-x^{b}\right) V_{b},
$$

where $V_{b}$ is the statistical volume associated with point $x^{b}$ as well as kernel function $W(x)$ and defined by

$$
V_{b}=\frac{1}{\sum_{a \in M_{b}} W\left(x^{b}-x^{a}\right)},
$$

where $M_{b}$ represents a set of particle points $x^{a}$ in the support of $W\left(x^{b}-x\right)$ for a given SPH discretization. The derivatives of $f(x)$ are evaluated simply by ordinary differentiation of Eq. (3). Generally, once a discretization is implemented according to Eq. (3), a dynamic problem could be solved by using difference scheme with respect to time.

Recently great efforts are devoted to solve the problems of instabilities, inaccuracies and implementation of boundary conditions on the surfaces of solids (Randles and Libersky, 1996; Chen et al., 1999; Chen and Beraun, 2000; Bonet and Kulasegaram, 2000, 2001; Liu et al., 2003c, etc.). Considerable progresses have been made in applications. However, there is still some weaknesses should be overcomed to realize its full potiential.

In the coming section, a very weak kernel estimate of linear isotropic elasto-dynamic system will be defined, followed by its discretization. In the fourth section, attention is focused on the boundary points, for which a modification of volumes, areas, and kernel functions is necessary with consideration of the local predefined feature of the boundaries. How to implement the boundary conditions, especially the transmitting boundaries is arranged in Section 5. In Section 6, there are discussions on stabilization by filtering and solution sharpening by de-convolution. Finally a numerical test is given to verify the effectiveness of the present method. 


\section{Very weak kernel estimate of elastodynamic system}

With the convention of summing on repeated coordinate indices adopted, the well known governing equation of a linear isotropic elastic wave system is

$$
\rho \ddot{u}_{i}(x, t)-\sigma_{i j, j}(x, t)=f_{i}(x, t), \quad t>0, x \in \Omega
$$

with the constitutive relation

$$
\sigma_{i j}=2 \mu \varepsilon_{i j}+\lambda \delta_{i j} \varepsilon_{k k}
$$

infinitesimal strain

$$
\varepsilon_{i j}=\frac{1}{2}\left(u_{i, j}+u_{j, i}\right)
$$

as well as the initial and boundary conditions

$$
\begin{array}{ll}
u_{i}(x, 0)=u_{i}^{0}, \quad \dot{u}_{i}(x, 0)=u_{i}^{1}, & x \in \Omega, \\
u_{i}(x, t)=g_{i}(x, t), \quad x \in \partial_{D} \Omega, & \sigma_{i j}(x, t) n_{j}=T_{i}(x, t), \quad x \in \partial_{N} \Omega,
\end{array}
$$

where $\partial_{D} \Omega$ and $\partial_{N} \Omega$ are the portion of the boundary of $\Omega$ with $\partial_{D} \Omega \oplus \partial_{N} \Omega=\partial \Omega, \rho, \lambda$ and $\mu$ are, respectively, the density and Lame constants of the medium in $\Omega$.

A very weak kernel estimate of the system is constructed by using the weighted residual method. Multiplying Eq. (5) by the kernel function concentrated at $x$ and integrating over the domain lead to

$$
\int \rho \ddot{u}_{i}(y, t) W(x-y) \mathrm{d} y-\int \sigma_{i j, j}(y, t) W(x-y) \mathrm{d} y=\int f_{i}(y, t) W(x-y) \mathrm{d} y .
$$

The second term in the left side of Eq. (10) can be integrated by parts as

$$
\int \sigma_{i j, j}(y, t) W(x-y) \mathrm{d} y=\int \sigma_{i j}(y, t) W_{, j}(x-y) \mathrm{d} y+\int_{\gamma} \sigma_{i j} n_{j} W(x-y) \mathrm{d} S_{y},
$$

where $\gamma$ is the intersection of the support of $W(x-y)$ and the boundary of $\Omega$. It should be noted that in Eq. (11), $W_{j}$ is the derivatives of $W$ with expression

$$
W_{, j}(x-y)=\frac{\partial}{\partial\left(x_{j}-y_{j}\right)} W(x-y)=-\frac{\partial}{\partial y_{j}} W(x-y) .
$$

Eq. (10) together with Eq. (11) is a weak kernel estimate of Eq. (5). Again by integrating by parts, the first term in the right side of Eq. (11) becomes

$$
\begin{aligned}
\int \sigma_{i j}(y, t) W_{, j}(x-y) \mathrm{d} y= & \int\left(2 \mu \varepsilon_{i j}(y, t)+\lambda \delta_{i j} \varepsilon_{k k}(y, t)\right) W_{, j}(x-y) \mathrm{d} y \\
= & \int\left(\mu \delta_{k i} W_{, j j}(x-y)+\mu W_{, k i}(x-y)+\lambda W_{, i k}(x-y)\right) u_{k}(y, t) \mathrm{d} y \\
& +\int_{\gamma}\left(\mu \delta_{k i} n_{j} W_{, j}(x-y)+\mu n_{i} W_{, k}(x-y)+\lambda n_{k} W_{, i}(x-y)\right) u_{k}(y, t) \mathrm{d} S_{y} .
\end{aligned}
$$

Analogous to Eq. (3) out of Eq. (1), Eq. (10) will result in

$$
\left\langle\rho \ddot{u}_{i}\right\rangle(x, t)-\left\langle f_{i}\right\rangle(x, t)-\left\langle T_{i}\right\rangle^{\gamma}(x, t)=\int K_{i k}(x-y) u_{k}(y, t) \mathrm{d} y+\int_{\gamma} \Gamma_{i k}(x-y) u_{k}(y, t) \mathrm{d} S_{y},
$$


where

$$
\begin{aligned}
& K_{i k}(x)=\mu \delta_{k i} W_{, j j}(x)+\mu W_{, k i}(x)+\lambda W_{, i k}(x), \\
& \Gamma_{i k}(x)=\mu \delta_{k i} n_{j} W_{, j}(x)+\mu n_{i} W_{, k}(x)+\lambda n_{k} W_{, i}(x)
\end{aligned}
$$

and

$$
\left\langle T_{i}\right\rangle^{\gamma}(x, t)=\int_{\gamma} W(x-y) T_{i}(y, t) \mathrm{d} S_{y}
$$

Eq. (14) is called as a very weak kernel estimate of Eq. (5). Compared with the weak kernel estimate (Liu, 2003), advantage of the former is that it contains only one integrating operation for each term whereas the latter contains double integrations. Such an advantage will lead to a considerable reduction of the computational cost.

\section{Discretization of the very weak kernel estimate}

Let $M_{N}=\left\{x_{n} ; n=1, \ldots, N\right\}$ represent the set of points forming an SPH discretization of $\bar{\Omega}=\Omega \cup \partial \Omega$ and $M_{b} \subset\{1, \ldots, N\}, b \in\{1, \ldots, N\}$, the set of particle points $x^{a}$ in the support of $W\left(x^{b}-x, h\right)$ for the given SPH discretization. As in Section 1, simplified notations by omitting $h$

$$
u_{i}^{a}(t)=u_{i}\left(x^{a}, t\right)
$$

and

$$
f_{i}^{a}(t)=f_{i}\left(x^{a}, t\right)
$$

will be used to express the discrete form of Eq. (14). The approximation of the third term in the left side of Eq. (14) is given by

$$
T_{i}^{a} \sum_{b \in M_{a}^{\gamma}} W_{a b} A_{b} \approx\left\langle T_{i}\right\rangle^{\gamma}\left(x^{a}, t\right)
$$

where $M_{a}^{\gamma}=\left\{b ; b \in M_{a}\right.$ and $\left.x^{b} \in \partial \Omega\right\}, W_{a b}=W\left(x^{a}-x^{b}\right)$ and $A_{b}$ is the statistical area of boundary point $x^{b}$ defined by

$$
A_{a}=\frac{1}{\sum_{b \in M_{a}^{\gamma}} S_{a} W_{a b}}
$$

where $S_{a}$ is selected to meet $\int_{\partial \Omega} S_{a} W\left(x^{a}-y\right) \mathrm{d} S_{y}=1$. The terms in the right side of Eq. (14) can be approximately written as

$$
\sum_{b \in M_{a}} K_{i k}^{a b} u_{k}^{b} V_{b}=\int K_{i k}\left(x^{a}-y\right) u_{k}(y, t) \mathrm{d} y
$$

and

$$
\sum_{b \in M_{a}^{\gamma}} \Gamma_{i k}^{a b} u_{k}^{b} A_{b}=\int \Gamma_{i k}\left(x^{a}-y\right) u_{k}(y, t) \mathrm{d} S_{y},
$$

where the summation convention for repeated indices works for the coordinate indices $i, j, k$, but not for the discretization labels $a, b$. 
For the left side of Eq. (14), using the central difference discretization with respect to time gives

$$
\rho \frac{u_{i}^{a}(t+\Delta t)-2 u_{i}^{a}(t)+u_{i}^{a}(t-\Delta t)}{\Delta t^{2}}-f_{i}^{a}(t)-T_{i}^{a}(t) \sum_{b \in M_{a}^{\gamma}} W_{a b} A_{b}=\sum_{b \in M_{a}} K_{i k}^{a b} V_{b} u_{k}^{b}(t)+\sum_{b \in M_{a}^{\gamma}} \Gamma_{i k}^{a b} A_{b} u_{k}^{b}(t) .
$$

\section{Volume and kernel-function modification for boundary points}

For the points in an SPH discretization near or on a boundary, the kernel estimations and the evaluations of the statistical volumes and areas will causes additional errors. In order to get a high accuracy, special treatments are needed.

For a boundary point, the influence of the boundary feature on the statistical volume can be represented by a scalar factor

$$
F_{a}^{V}=\frac{\text { Volume of the intesection of the support of } W \text { and } \Omega}{\text { Volume of the support of } W},
$$

given a pre-treatment. Obviously, $F_{a}^{V}=1$ for an inner point $x^{a}$ far from the boundary. The modified statistical volume can be defined as

$$
V_{a}=\frac{F_{a}^{V}}{\sum_{b \in M_{a}} W_{a b}} .
$$

Analogously, the influence of the boundary feature on the statistical area can be represented by a scalar factor defined by

$$
F_{a}^{A}=\frac{\text { Area of the intesection of the support of } W \text { and } \partial \Omega}{\text { Area of the intesection of the support of } W \text { and a plan tangential of } \partial \Omega},
$$

then the modified statistical area will be

$$
A_{a}=\frac{F_{a}^{A}}{\sum_{b \in M_{a}^{\gamma}} S_{a} W_{a b}} .
$$

If the smoothing length $h$ is not too large compared with to the distance between the particle points in the discretization, the modifications are only needed for boundary points. For boundary point $x^{a}$, function $W\left(x_{a}-x_{b}\right)$ should be modified by

$$
\widehat{W}\left(x_{a}-x_{b}\right)=\alpha\left(x_{a}\right) W\left(x_{a}-x_{b}\right),
$$

where

$$
\alpha\left(x_{a}\right)=\frac{1}{\sum_{b \in M_{a}} W\left(x_{a}-x_{b}\right) \cdot V_{b}} .
$$

\section{About boundary conditions}

Randles and Libersky (1996) pointed out that boundary conditions in SPH have been both a sore point and a neglected subject. The early fluid dynamic problems solved with SPH only possess very simple boundary condition even no boundary condition to be treated. In early interpolating particle code of SPH, the boundary or edge of a domain was never very well defined. How to precisely prescribe the boundary 
and how to improve the deficient kernel summation at boundary are two major problems in boundary condition treatment of SPH method.

In our study, the boundary is described by boundary particles, and each boundary particle has a local geometrical factor defined by Eq. (24) to determine the volume ratio, and the kernel sum deficiencies at boundary are overcome by modified statistical volumes and kernel functions as described in Section 4. The approximated boundary condition

$$
u_{i}^{a}(t)=\left\langle g_{i}\right\rangle\left(x^{a}, t\right)=\sum_{b \in M_{a}^{\gamma}} g_{i}^{b}(t) W_{a b} S_{a} A_{a} \quad \text { for all } t>0
$$

can be used to deal with the points on boundary with known displacement in Eq. (23).

Transmitting boundaries have to be considered for semi-infinite or infinite domains that often appear in the simulation of seismic wave propagations.

The transmitting or silent boundary can be determined by collecting a complete list of boundary points. In the approach used by Cohen and Jennings (1983), who in turn credited the method to Lysmer and Kuhlemeyer, viscous normal stress and shear stresses are exerted on the boundary points in the form

$$
\begin{aligned}
& \sigma_{\text {normal }}=-\rho c_{\mathrm{p}} V_{\text {normal }}, \\
& \sigma_{\text {shear }}=-\rho c_{\mathrm{s}} V_{\text {tan } g},
\end{aligned}
$$

where $\rho, c_{\mathrm{p}}$ and $c_{\mathrm{s}}$ are the density, the dilatational wave speed, and the shear wave speed of the medium, respectively. The magnitudes of the stresses are proportional to the particle velocity separately in the normal direction, $V_{\text {normal }}$ and tangential direction, $V_{\tan g}$. The vector form of the stress in a local coordinate system could be expressed as

$$
T_{\mathrm{loc}}=-\rho C v_{\mathrm{loc}}
$$

where

$$
T_{\text {loc }}=\left(\begin{array}{c}
\sigma_{\text {normal }} \\
\sigma_{\text {shear } 1} \\
\sigma_{\text {shear } 2}
\end{array}\right), \quad C=\left[\begin{array}{ccc}
c_{\mathrm{p}} & 0 & 0 \\
0 & c_{\mathrm{s}} & 0 \\
0 & 0 & c_{\mathrm{s}}
\end{array}\right], \quad V_{\text {loc }}=\left(\begin{array}{c}
V_{\text {normal }} \\
V_{\text {tang1 }} \\
V_{\text {tang2 }}
\end{array}\right) .
$$

Let $R_{a}$ be the rotation matrix from the local coordinate system of boundary point $x^{a}$ to the global Cartesian coordinate system, then the transmitting boundary condition could be added into the governing equation. For the particle points on transmitting boundary, Eq. (23) should be replaced with

$$
\begin{aligned}
& \rho \frac{U_{\mathrm{loc}}^{a}(t+\Delta t)-2 U_{\mathrm{loc}}^{a}(t)+U_{\mathrm{loc}}^{a}(t-\Delta t)}{\Delta t^{2}}-R_{a}^{T} f_{i}^{a}(t)-C V_{\mathrm{loc}}^{a}(t) \sum_{b \in M_{a}^{\gamma}} W_{a b} A_{b} \\
&=R_{a}^{T} \sum_{b \in M_{a}} K_{i k}^{a b} V_{b} u_{k}^{b}(t)+R_{a}^{T} \sum_{b \in M_{a}^{\gamma}} \Gamma_{i k}^{a b} A_{b} u_{k}^{b}(t)
\end{aligned}
$$

with

$$
V_{\mathrm{loc}}^{a}(t)=\frac{U_{\mathrm{loc}}^{a}(t+\Delta t)-R_{a}^{T} U^{a}(t-\Delta t)}{2 \Delta t}
$$

and

$$
U^{a}(t)=\left(\begin{array}{l}
u_{1}^{a}(t) \\
u_{2}^{a}(t) \\
u_{3}^{a}(t)
\end{array}\right)
$$


The displacement vector can be transformed with

$$
U^{a}(t+\Delta t)=R_{a} U_{\mathrm{loc}}^{a}(t+\Delta t) .
$$

\section{Stabilization and solution sharpening}

To avoid the tensile instability and the spurious modes, the modified Balsala's filter (Randles and Libersky, 1996)

$$
U_{\mathrm{ef}}^{a}=U^{a}+\alpha\left[\frac{\sum_{b \neq a} U^{b} W_{a b} V_{b}}{\sum_{b \neq a} U W_{a b} V_{b}}-U^{a}\right]
$$

can be used. When $\alpha$ is relatively small, it does not cause significant additional errors.

Solution of Eq. (23) or/and Eq. (33) is a smoothed result of the solution of Eq. (5) because of the kernel estimation. Therefore, for relatively coarse distribution of the particle points, the numerical solution will have a considerable error. To improve the numerical result, an a correction by de-convolution should be carried out.

From

$$
\left\langle u_{i}\right\rangle(x, t)=\int u_{i}(y, t) W(x-y, h) \mathrm{d} y \approx \sum_{b} u_{i}\left(x^{b}, t\right) W\left(x-x^{b}\right) V_{b},
$$

it is known that

$$
u_{i}\left(x^{a}, t\right) \approx \frac{\left\langle u_{i}\right\rangle\left(x^{a}, t\right)-\sum_{b \neq a} u_{i}\left(x^{b}, t\right) W\left(x^{a}-x^{b}\right) V_{b}}{W(0) V_{a}} .
$$

Replacing $u_{i}\left(x^{b}, t\right)$ with $u_{i}^{h}\left(x^{b}, t\right)$ for adjacent points of $x^{a}$ in Eq. (37) gives

$$
u_{i}\left(x^{a}, t\right) \approx \frac{\left\langle u_{i}\right\rangle(x, t)-\sum_{b \neq a}\left\langle u_{i}\right\rangle\left(x^{b}, t\right) W_{a b} V_{b}}{W(0) V_{a}} .
$$

Eq. (38) is able to convert the weighted numerical solution to a more accurate result.

\section{Numerical tests}

In the following example, the cubic spline kernel function (Randles and Libersky, 1996)

$$
W(v, h)=\frac{1}{\pi h^{3}} \begin{cases}\frac{3}{2}\left(\frac{2}{3}-v^{2}+\frac{1}{2} v^{3}\right) & 0 \leqslant v<1, \\ \frac{1}{4}(2-v)^{3} & 1 \leqslant v \leqslant 2 \\ 0 & v \geqslant 2\end{cases}
$$

where

$$
v=\frac{r}{h}=\frac{\left|x_{b}-x_{a}\right|}{h}
$$

is employed to simulate the elastic wave propagation in a half space in $R^{3}$. It can be verified that

$$
S_{a}=\frac{10 h}{7} \text { for all } a
$$


The calculation is performed on a hexahedron with dimension $7 \times 7 \times 7$. On each face of the hexahedron, the transmitting boundary conditions are imposed except the one defined by $x_{3}=0$, on the center of which an impulsive load in the form:

$$
T_{z}(x, t)=f(t) X\left(x_{1}, x_{2}\right) \quad x=\left(x_{1}, x_{2}, x_{3}\right)
$$

with

$$
f(t)=\left\{\begin{array}{ll}
t & 0 \leqslant t<1, \\
2-t & 1 \leqslant t<2, \\
0 & \text { otherwise }
\end{array} \text { and } \quad X\left(x_{1}, x_{2}\right)= \begin{cases}1 & \left|x_{1}-3.5\right| \leqslant 0.5 \text { and }\left|x_{2}-3.5\right| \leqslant 0.5 \\
0 & \text { otherwise }\end{cases}\right.
$$



Fig. 1. The distribution of the $15 \times 15 \times 15$ particles (shaded picture).



Fig. 2. Vertical velocity at the center of the load area. 


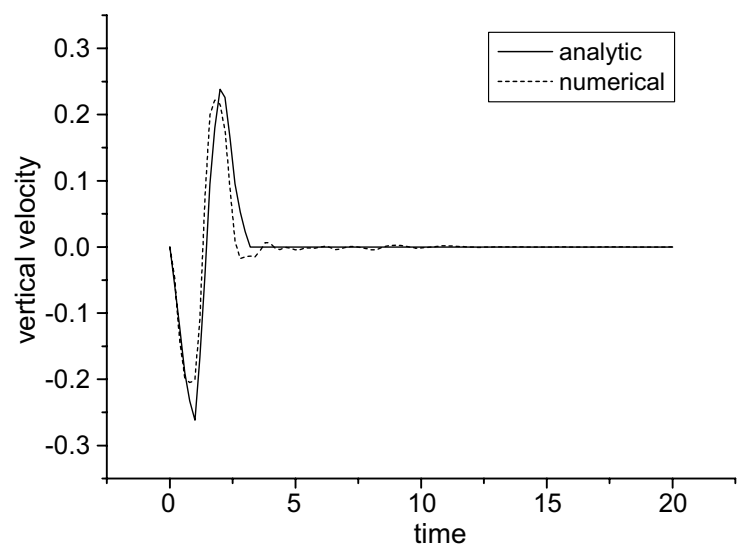

Fig. 3. Vertical velocity at a point of free surface out of the load area.

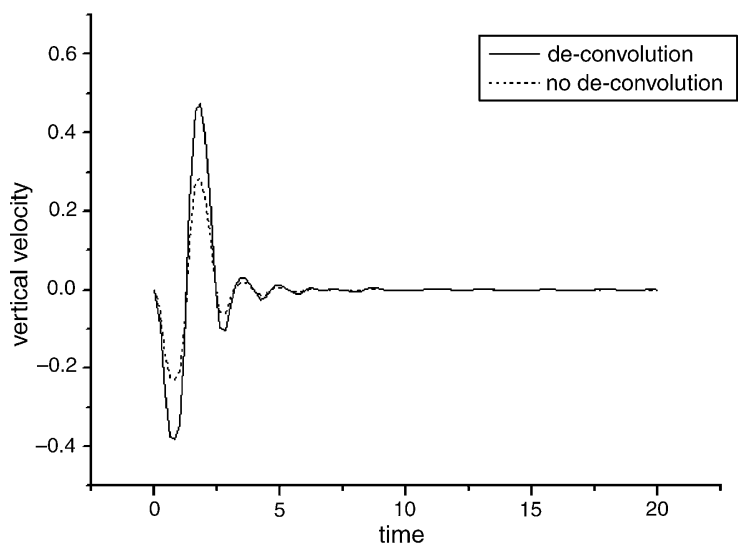

Fig. 4. The illustration of the improvement of the solution by de-convolution (vertical velocity at the center of the load area).

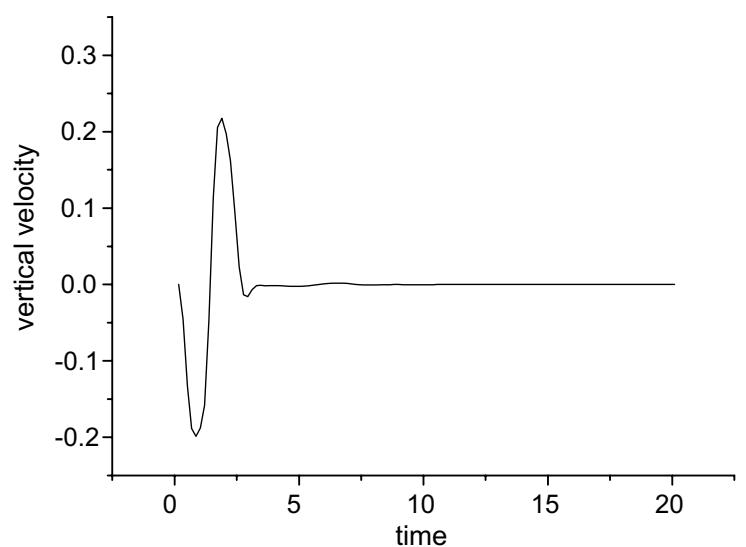

Fig. 5. Numerical result of vertical velocity at the center of the load with $\alpha=0.5$. 
is imposed, whereas the other part is stress free. The model problem has elastic constants $\lambda=\mu=1$ and density $\rho=1$. The numerical result is obtained with $15 \times 15 \times 15=3375$ particle points which are uniformly distributed in the hexahedron, so the distance of two neighboring particles is 0.5 (see Fig. 1). In the calculation, $\alpha$ in Eq. (35) is taken as 0.1 and the results are converted by Eq. (38).

Figs. 2 and 3 show that the numerical result is favorably compared with the analytic solution. The conversion of the solution by de-convolution defined in Eq. (38) can greatly improve the accuracy for a coarse distribution of particles(see Fig. 4).

When $\alpha$ gets relatively large, e.g. $\alpha=0.5$, the velocity curve shown in Fig. 5 is more smooth but its amplitude diminish. The accuracy will be improved when the distribution particles is refined in the discretization.

\section{Conclusions}

The primary simulation of the elastic wave propagation with the present smoothed point method indicates that the method and the treatments of transmitting boundary condition proposed work properly. It is verified that the conversion of the solution by de-convolution allows to use relatively large particle distance. Compared with the traditional SPH method, the modifications of statistical volumes for the boundary points and their kernel functions improve the accuracy significantly, but only cause little additional computational cost.

\section{Acknowledgement}

This work is supported by The Special Funds for Major State Basic Research Project under Grant No. 2002CB412706 and the project: "The crucial mechanical problems in landsliding engineering" of Chinese Academy of Sciences.

\section{References}

Bonet, J., Kulasegaram, S., 2000. Correction and stabilization of smooth particle hydrodynamics methods with applications in metal forming simulations. International Journal for Numerical Methods in Engineering 47, 1189-1214.

Bonet, J., Kulasegaram, S., 2001. Remarks on tension instability of Eulerian and Lagrangian corrected smooth particle hydrodynamics (CSPH) methods. International Journal for Numerical Methods in Engineering 52, 1203-1220.

Chen, J.K., Beraun, J.E., 2000. A generalized smoothed particle hydrodynamics method for nonlinear dynamic problems. Computer Methods in Applied Mechanics and Engineering 19, 225-239.

Chen, J.K., Beraun, J.E., Jih, C.J., 1999. Completeness of corrective smoothed particle method for linear elastodynamics. Computational Mechanics 24, 273-285.

Cohen, M., Jennings, P.C., 1983. Silent boundary methods for transient analysis. In: Belytschko, T., Hughes, T.J.R. (Eds.), Computational Methods for Transient Analysis. North-Holland, New York, pp. 301-360.

Gingold, R.A., Monaghan, J.J., 1977. Smoothed particle hydrodynamics: theory and application to non-spherical stars. Monthly Notices of the Royal Astronomical Society 181, 375-389.

Libersky, L.D., Petschek, A.G., 1991. Smoothed particle hydrodynamics with strength of materials. In: Trease, H., Fritts, J., Crowley, W. (Eds.), Proceedings, The Next Free Lagrange Conference, vol. 395. Springer-Verlag, NY, pp. 248-257.

Liu, G.R., 2003. Mesh Free Methods: Moving Beyond the Finite Element Method. CRC Press.

Liu, M.B., Liu, G.R., Lam, K.Y., 2002. Investigations into water mitigations using a meshless particle method. Shock Waves 12 (3), $181-195$.

Liu, G.R., Liu, M.B., 2003. Smoothed Particle Hydrodynamics, A Meshfree Particle Method. World Scientific.

Liu, M.B., Liu, G.R., Lam, K.Y., 2003a. Constructing smoothing functions in smoothed particle hydrodynamics with applications. Journal of Computational and Applied Mathematics 155 (2), 263-284.

Liu, M.B., Liu, G.R., Lam, K.Y., 2003b. Meshfree particle simulation of the explosion process for high explosive in shaped charge. Shock Waves $12(6), 509-520$. 
Liu, M.B., Liu, G.R., Zong, Z., Lam, K.Y., 2003c. Computer simulation of high explosive explosion using smoothed particle hydrodynamics methodology. Computer and Fluids 32, 305-322.

Liu, M.B., Liu, G.R., Zong, Z., Lam, K.Y., 2003d. Smoothed particle hydrodynamics for numerical simulation of underwater explosions. Computational Mechanics 30 (2), 106-118.

Lucy, L.B., 1977. A numerical approach to the testing of fusion process. The Astronomical Journal 82, $1013-1024$.

Owen, J.M., Villumsen, J.V., Shapiro, P.R., Martel, H., 1998. Adaptive smoothed particle hydrodynamics methodology ii. Astrophysical Journal Supplement Series 116, 155-209.

Randles, P.W., Libersky, L.D., 1996. Smoothed particle hydrodynamics: some recent improvements and applications. Computer Methods in Applied Mechanics and Engineering 139, 375-408.

Randles, P.W., Libersky, L.D., 2000. Normalized SPH with stress point. International Journal for Numerical Methods in Engineering 47, 1445-1462. 